\title{
polymers
}

ISSN 2073-4360

www.mdpi.com/journal/polymers

Article

\section{Radical-Scavenging Activity of Thiols, Thiobarbituric Acid Derivatives and Phenolic Antioxidants Determined Using the Induction Period Method for Radical Polymerization of Methyl Methacrylate}

\section{Yoshinori Kadoma $^{1, *}$ and Seiichiro Fujisawa ${ }^{2}$}

1 Institute of Biomaterials and Bioengineering, Tokyo Medical and Dental University, Kanda-surugadai, Chiyoda-ku, Tokyo 101-0062, Japan

2 Meikai University School of Dentistry, Sakado, Saitama 350-0283, Japan;

E-Mail: fujisawa33@nifty.com

* Author to whom correspondence should be addressed; E-Mail: y-kadoma.fm@tmd.ac.jp; Tel.: +81-3-5280-8030; Fax: +81-3-5280-8005.

Received: 27 February 2012; in revised form: 2 April 2012 / Accepted: 5 April 2012 /

Published: 16 April 2012

\begin{abstract}
The radical-scavenging activities of two thiols, eight (thio)barbituric acid derivatives and six chain-breaking phenolic antioxidants were investigated using the induction period method for polymerization of methyl methacrylate (MMA) initiated by thermal decomposition of 2,2'-azobisisobutyronitrile (AIBN) and monitored by differential scanning calorimetry (DSC). The induction period (IP) for the thiols 2-mercaptoethanol (ME) and 2-mercapto-1-methylimidazole (MMI) was about half that for phenolic antioxidants. Except for the potent inhibitor 5,5-dimethyl-2-thiobarbituric acid (3), the IP for thiobarbituric acid derivatives was about one tenth of that for phenolic antioxidants. The IP for 1,3,5-trimethyl-2-thiobarbituric acid (1) and 5-allyl-1, 3-dimethyl-2-thiobarbituric acid (7) was less than that of the control, possibly due to inhibition by a small amount of atmospheric oxygen in the DSC container. The ratio of the chain inhibition to that of chain propagation $(\mathrm{CI} / \mathrm{CP})$ for the thiols and thiobarbituric acid compounds except for $\mathbf{1}, \mathbf{3}$ and $\mathbf{7}$ was about 10 times greater or greater than that for phenolic compounds. A kinetic chain length (KCL) about $10 \%$ greater than that of the control was observed for $\mathbf{1}$, suggesting that $\mathbf{1}$ had chain transfer reactivity in the polymerization of MMA. The average molecular weight of polymers formed from thiobarbituric acid derivatives is discussed.
\end{abstract}


Keywords: organic sulfur compounds; phenolic compounds; radical polymerization; AIBN;

MMA; induction period; initial rate of polymerization; chain inhibition; chain propagation

\section{Introduction}

Quantitative inhibition of auto-oxidation by various synthetic and natural phenols and organic sulfur compounds has been investigated by many researchers [1-9]. Burton and Ingold studied the radical-scavenging activity of various phenolic compounds using the induction period method in a chlorobenzene-styrene system initiated by AIBN, and found that phenolic compounds act as potent chain-breaking antioxidants [1]. Pryor et al. investigated the radical-scavenging activity of various antioxidants for the peroxy radical from linoleic acid-SDS (sodium dodecyl sulfate) micelles at $37{ }^{\circ} \mathrm{C}$ and found that phenolic compounds such as vitamin $\mathrm{E}$ were potent antioxidants, whereas the radical-scavenging activities of beta-carotene (a polyene), meladione (a quinone), aminopyrine (a tertiary amine), and ibuprofen (a benzylacetic acid derivative) were undetectable because their induction periods were too small to be measurable in their system [2]. In contrast, the radical-scavenging activity of 1,3-dimethylthiourea was reported to be detectable at higher oxygen tensions, although this compound had lower radical-scavenging activity [2]. Both chlorobenzene-styrene [1] and linoleic acid-aqueous systems [2] have been investigated under fully oxygenated conditions, and therefore some of the compounds described above were not evaluable due to their interaction with oxygen as a biradical. Previously, we investigated the radical-scavenging activity of the reaction products of isoeugenol (2-methoxy-4-propenylphenol) with thiol, thiophenol, mercaptothiazoline or mercaptomethylimidazole using the induction period method for polymerization of MMA initiated by thermal decomposition of AIBN under nearly anaerobic conditions. The reaction products of isoeugenol with an SH group showed higher inhibition rate constants (kinh) than the parent isoeugenol. In fact, even at relatively small concentrations, these products enhanced the initial rate of polymerization (IRP) value by about $10 \%$ comparing to that of relative to control, suggesting possible chain transfer reactivity for these sulfur compounds [3]. We have previously synthesized thiobarbituric acid derivatives such as 1,3,5-trimethyl2-thiobarbituric acid (1), 1,5,5-trimethyl-2-thiobarbituric acid (2), 5,5-dimethyl-2-thiobarbituric acid (3), 5-butyl-2-thiobarbituric acid (4), 5-butylbarbituric acid (5), 5-allyl-2-thiobarbituric acid (6), 5-allyl-1,3-dimethyl-2-thiobarbituric acid (7) and 5-(4-vinylbenzyl)-2-thiobarbituric acid (8) (see Table 1), and investigated their radical-scavenging activity using the induction period method for polymerization of MMA initiated by thermal decomposition of AIBN [4,5]. However, there have been few kinetic studies of these compounds. Also, thiols such as 2-mercaptoethanol and 2-mercaptothioxanthone have been previously reported to act as chain transfer agents in radical polymerization [6,7]. The high efficiency of organic sulfur compounds for control of chain length has attracted considerable attention in the field of radical polymerization, since the mechanical properties of the polymers obtained are dependent on their molecular weight.

In the present study, in the light of currently available data, we investigated the radical-scavenging activities of two thiols (2-mercaptoethanol, 2-mercapto-1-methylimidazole), eight (thio)barbituric acid derivatives (compounds 1-8) and six chain-breaking phenolic antioxidants ( $p$-cresol, eugenol, 
hydroquinone, phenol, thymol, $\alpha$-tocopherol) using the induction period method for polymerization of MMA initiated by thermal decomposition of AIBN. Also, the influence of thiobarbituric acid derivatives on the average molecular weight of the polymers formed from MMA was discussed.

\section{Experimental Section}

\subsection{Materials}

Thiobarbituric acid derivatives, compounds 1-8, were synthesized from diethyl malonates [4,5], see Scheme 1.

Scheme 1. Structure of the thiobarbituric acid derivative.

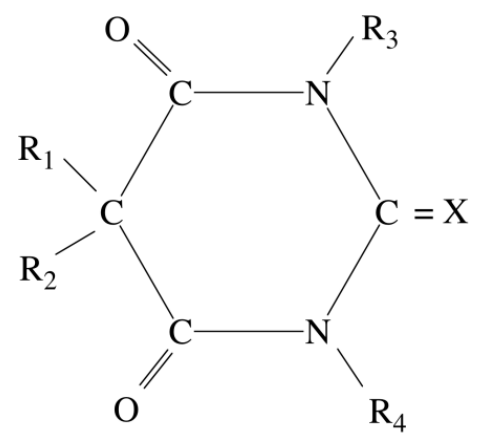

Table 1. The thiobarbituric acid derivatives used and their structures.

\begin{tabular}{|c|c|c|c|c|c|}
\hline Compound $^{\mathrm{a}}$ & $\mathbf{R}_{1}$ & $\mathbf{R}_{2}$ & $\mathbf{R}_{3}$ & $\mathbf{R}_{4}$ & $\mathbf{X}$ \\
\hline 1 & $\mathrm{CH}_{3}$ & $\mathrm{H}$ & $\mathrm{CH}_{3}$ & $\mathrm{CH}_{3}$ & $\mathrm{~S}$ \\
\hline 2 & $\mathrm{CH}_{3}$ & $\mathrm{CH}_{3}$ & $\mathrm{CH}_{3}$ & $\mathrm{H}$ & $\mathrm{S}$ \\
\hline 3 & $\mathrm{CH}_{3}$ & $\mathrm{CH}_{3}$ & $\mathrm{H}$ & $\mathrm{H}$ & $\mathrm{S}$ \\
\hline 4 & $\mathrm{CH}_{3} \mathrm{CH}_{2} \mathrm{CH}_{2} \mathrm{CH}_{2}-$ & $\mathrm{H}$ & $\mathrm{H}$ & $\mathrm{H}$ & $\mathrm{S}$ \\
\hline 5 & $\mathrm{CH}_{3} \mathrm{CH}_{2} \mathrm{CH}_{2} \mathrm{CH}_{2-}$ & $\mathrm{H}$ & $\mathrm{H}$ & $\mathrm{H}$ & $\mathrm{O}$ \\
\hline 6 & $\mathrm{H}_{2} \mathrm{C}=\mathrm{CH}-\mathrm{CH}_{2}-$ & $\mathrm{H}$ & $\mathrm{H}$ & $\mathrm{H}$ & $\mathrm{S}$ \\
\hline 7 & $\mathrm{H}_{2} \mathrm{C}=\mathrm{CH}-\mathrm{CH}_{2}-$ & $\mathrm{H}$ & $\mathrm{CH}_{3}$ & $\mathrm{CH}_{3}$ & $\mathrm{~S}$ \\
\hline 8 & $\mathrm{H}_{2} \mathrm{C}=\mathrm{CH}-\mathrm{C}_{6} \mathrm{H}_{5}-\mathrm{CH}_{2}-$ & $\mathrm{H}$ & $\mathrm{H}$ & $\mathrm{H}$ & $\mathrm{S}$ \\
\hline
\end{tabular}

The following chemicals and reagents were obtained from the indicated companies: ME, MMI, phenolic compounds ( $\alpha$-tocopherol, guaiacol, eugenol, thymol, $p$-cresol, phenol, 2,6-di-tert-butyl-4-methoxyphenol), MMA (Tokyo Kasei Kogyo, Co., Ltd., Tokyo, Japan). MMA was purified by distillation. AIBN (Wako Pure Chemical Industries Ltd. Japan) was recrystallized from chloroform. The chemical structure of MMI is shown in Scheme 2.

Scheme 2. Structure of 2-mercapto-1-methylimidazole.<smiles>Cn1ccnc1S</smiles> 


\subsection{Induction Period (IP) and Initial Rate of Polymerization (IRP)}

The IP and IRP values in the presence $\left(R p_{\text {inh }}\right)$ or absence $\left(\mathrm{Rp}_{\mathrm{con}}\right)$ of an antioxidant were determined by the method reported previously [3,9]. In brief, the experimental resin consisted of MMA and AIBN with or without additives. AIBN was added at $1.0 \mathrm{~mol} \%$, and the additives were used at $0.01,0.02$, 0.05 or $1 \mathrm{~mol} \%$. Approximately $10 \mu \mathrm{L}$ of the experimental resin (MMA: 9.15-9.30 mg) was loaded into an aluminum sample container and sealed by applying pressure. The container was placed in a DSC (model DSC 3100; Mac Science Co., Tokyo, Japan) kept at $70{ }^{\circ} \mathrm{C}$, and the thermal changes induced by polymerization were recorded for the appropriate periods. The heat due to the polymerization of MMA was $13.0 \mathrm{kcal} \mathrm{mol}^{-1}$. The conversion of all samples (\%) was calculated from the DSC thermograms using the integrated heat evoked by polymerization of MMA. The value was 92.5-95.8\%. The time-exotherm and time-conversion curves are shown in Figure 1. Time-conversion curves break when an inhibitor is consumed. These breaks are sharp and provide a reliable measure of the induction time of the inhibitor. The IP was calculated from the difference between the IP of the inhibitors and that of the controls. The IRP values in the absence $\left(\mathrm{Rp}_{\mathrm{con}}\right)$ and presence $\left(\mathrm{Rp}_{\mathrm{inh}}\right)$ of an inhibitor were calculated from the slope of the plots during the initial linear phase of MMA polymerization (tangent drawn at the early polymerization stage), as reported previously [3,9].

\subsection{Measurement of Stoichiometric Factor (n)}

The $n$ value in Equation (1) can be calculated from the induction period (time) in the presence of inhibitors:

$$
n=\mathrm{R}_{\mathrm{i}}[\mathrm{IP}] /[\mathrm{IH}]
$$

where IP is the induction period in the presence of an inhibitor (IH). The number of moles of AIBN radicals trapped by the antioxidant was calculated with respect to 1 mole of the inhibitor moiety. The initiation rate $\left(\mathrm{R}_{\mathrm{i}}\right)$ for AIBN at $70{ }^{\circ} \mathrm{C}$ was $6.24 \times 10^{-6} \mathrm{~mol} \mathrm{l}^{-1} \mathrm{~s}^{-1}$, calculated on the basis of $n=2.0$ for 2, 6-di-tert-butyl-4-methoxyphenol.

\subsection{Measurement of the Inhibition Rate Constant $\left(k_{i n h}\right)$}

When $R_{i}$ is constant, i.e., when new chains are initiated at a constant rate, a steady-state treatment can be applied and the initial rate of polymerization of MMA is given by Equation (2):

$$
\mathrm{Rp}_{\mathrm{con}}=\left(\mathrm{k}_{\mathrm{p}}[\mathrm{MMA}] \mathrm{R}_{\mathrm{i}}^{1 / 2}\right) /\left(2 \mathrm{k}_{\mathrm{t}}\right)^{1 / 2}
$$

where MMA represents methyl methacrylate and $\mathrm{k}_{\mathrm{p}}$ and $\mathrm{k}_{\mathrm{t}}$ are the rate constants for chain propagation and termination, respectively. The $R p_{\text {inh }}$ rates are determined by Equation (3):

$$
\mathrm{Rp}_{\text {inh }}=\left(\mathrm{k}_{\mathrm{p}}[\mathrm{MMA}] \mathrm{R}_{\mathrm{i}}\right) /\left(n \mathrm{k}_{\mathrm{inh}}[\mathrm{IH}]\right)
$$

where $\mathrm{Rp}_{\mathrm{inh}}$ is the initial rate of inhibited polymerization, [MMA], $n,[\mathrm{IH}]$ and $\mathrm{k}_{\mathrm{p}}$ are defined above, and $\mathrm{k}_{\mathrm{inh}}$ is the rate constant for scavenging (inhibition) of MMA radicals by an antioxidant. From Equation 2 and Equation 3, $\mathrm{k}_{\mathrm{inh}} / \mathrm{k}_{\mathrm{p}}$ can be calculated using Equation (4): 


$$
\mathrm{k}_{\mathrm{inh}} / \mathrm{k}_{\mathrm{p}}=[\mathrm{MMA}] /\left(\mathrm{Rp}_{\mathrm{inh}}[\mathrm{IP}]\right)
$$

The ratio of the rate of chain inhibition to that of chain propagation $(\mathrm{CI} / \mathrm{CP})$ was determined using Equation (5).

$$
\mathrm{CI} / \mathrm{CP}=\left(\mathrm{k}_{\mathrm{inh}}\left[\mathrm{R}^{*}\right][\mathrm{IH}]\right) /\left(\mathrm{k}_{\mathrm{p}}\left[\mathrm{R}^{*}\right][\mathrm{MMA}]\right)=\left(\mathrm{k}_{\mathrm{inh}}[\mathrm{IH}]\right) /\left(\mathrm{k}_{\mathrm{p}}[\mathrm{MMA}]\right)=[\mathrm{IH}] /\left(\mathrm{Rp}_{\mathrm{inh}}[\mathrm{IP}]\right)
$$

where $\mathrm{R}^{*}$ represents radicals derived from thermal decomposition of AIBN.

The kinetic chain length (KCL) was determined using Equation (6).

$$
\mathrm{KCL}=\mathrm{IRP} / \mathrm{R}_{\mathrm{i}}
$$

where IRP is the initial rate of polymerization $(\mathrm{Rp})$.

\subsection{Determination of Molecular Weight}

The average molecular weight of polymer formed for thiobarbituric acid derivatives was taken from our data reported previously [4,5]. Briefly, after polymerization for $100 \mathrm{~h}$, the polymer formed was reprecipitated in methanol. Poly(methyl methacrylate) was dissolved in ethyl acetate and then number average molecular weight $(\mathrm{Mn})$ and weight average molecular weight $(\mathrm{Mw})$ were determined using gel permeation chromatography (GPC) (GC-9AM, Shimadzu Co. Osaka, Japan) with a DOP 2m column.

The polydispersity index (PI) was defined by the following Equation (7) [10]:

$$
\mathrm{PI}=\mathrm{Mw} / \mathrm{Mn}
$$

\section{Results and Discussion}

\subsection{Thiol}

We investigated the radical-scavenging activity of $\mathrm{ME}$ at a relatively low concentration of 0.01-0.05 mol\%, and one example of its results is shown in Table 2 and Figure 1, respectively.

Table 2. Polymerization of methyl methacrylate (MMA) in the presence of 2-mercaptoethanol (ME).

\begin{tabular}{llllll}
\hline Additives & $\begin{array}{l}\text { Induction } \\
\text { period } \\
(\mathbf{m i n})\end{array}$ & $\begin{array}{l}\text { Initial rate } \\
\text { polymerization } \\
(\boldsymbol{\%} / \mathbf{m i n})\end{array}$ & $\begin{array}{l}\text { of Time at maximum Maximum } \\
\text { exothermic peak }\end{array}$ & $\begin{array}{l}\text { exothermic rate } \\
\text { (mcal/min) }\end{array}$ & $\begin{array}{l}\text { Conversion } \\
(\boldsymbol{\%})\end{array}$ \\
\hline None & 3.38 & 1.321 & 44.18 & 207.54 & 95.9 \\
ME $(0.01 \mathrm{~mol} \%)$ & 4.19 & 1.340 & 41.85 & 200.13 & 93.3 \\
\hline
\end{tabular}

The induction period for ME was larger than that for the control. The time at the maximum exothermic peak and the maximum exothermic rate for $\mathrm{ME}$ were smaller than the corresponding values for the control. $\mathrm{ME}$ (structure: $\mathrm{HS}-\mathrm{CH}_{2} \mathrm{CH}_{2} \mathrm{OH}$ ) at the indicated concentrations acted as an inhibitor due to $\mathrm{H}$ abstraction from the -SH group in the ME molecule by free radicals (Figure 2). The weakness of the S-H bond and the high reactivity of the thiyl radical (RS*) are the two crucial parameters for a chain transfer agent $[7,8]$. Nucleophilic thiyl radicals are expected to be more reactive toward the $\alpha, \beta$-unsaturated carbonyl group in the MMA molecule because of their higher electrophilicity. If MMA was polymerized by thiyl radicals derived from ME, then sulfur compounds 
would be detected in the polymers formed. Okada et al. investigated the polymerization of MMA in the presence of both ME and AIBN, and found that no sulfur compounds were present in the polymers obtained [11]. This suggested that the polymerization of MMA did not involve thiyl radicals.

Figure 1. Exothermic (upper panel) and time-conversion (lower panel) curves for the polymerization of MMA with 2,2'-azobisisobutyronitrile (AIBN) in the presence of $0.01 \mathrm{~mol} \%$ 2-mercaptoethanol.
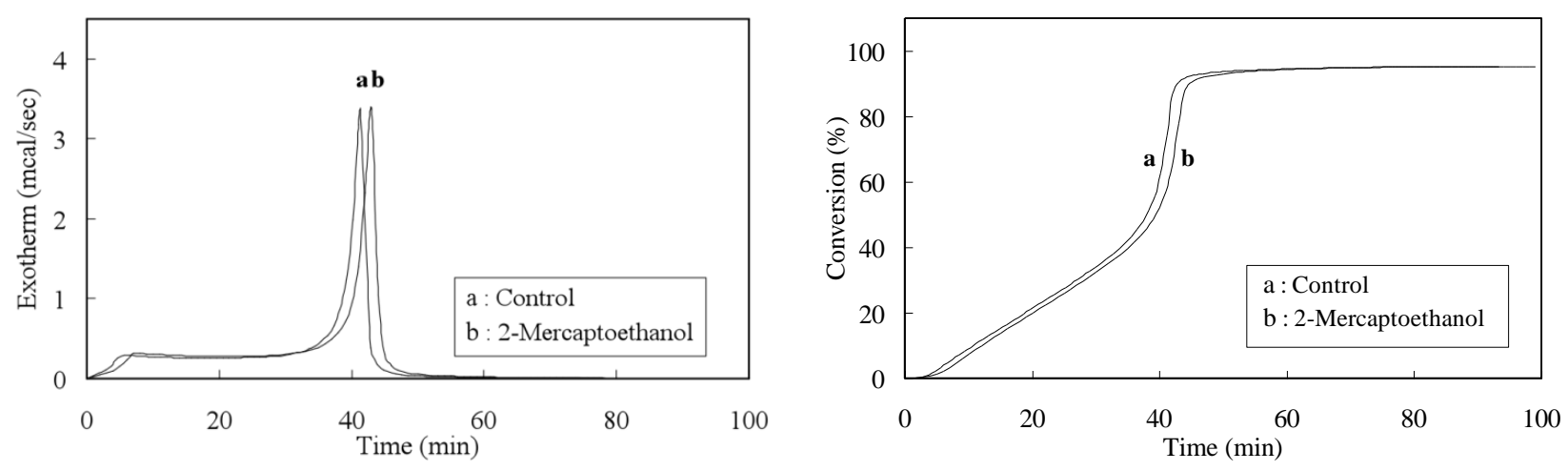

Figure 2. The reaction of a thiol with a poly(MMA) growing radical *.<smiles>[R][C@H](C)[C@H](C)C(=O)OC</smiles>

The chain transfer constant of ME for growing poly(MMA) radicals is reported to be 0.62 , although the value for aliphatic thiols such as ME is smaller than that for aromatic thiols [7]. This indicates that for a higher concentration of $\mathrm{ME}$, the chain transfer reaction of this compound could occur during polymerization of MMA. It has been reported that for thermal polymerization of MMA in the presence of thioxanthone, an aromatic thiol initiated by AIBN, thioxanthone acted as a chain transfer agent when 1-10 $\mathrm{mM}$ thioxanthone was added to $1 \mathrm{mM}$ AIBN. This compound is thermally stable and does not initiate itself in the absence of AIBN. The addition of a millimolar concentration of this compound to the polymerization of both styrene and MMA decreased the molecular weight of the polymer formed [8].

The result for 2-mercapto-1-methylimidazole (MMI) is also shown in Table 2. The radical-scavenging activity of MMI was similar to that of ME. We investigated for how long and how efficiently ME and MMI suppressed chain propagation during polymerization of MMA in the AIBN system. The ratio of the rate of chain inhibition to that of chain propagation was determined using Equation (5). The $\mathrm{CI} / \mathrm{CP}$ values for $\mathrm{ME}$ and $\mathrm{MMI}$ in the concentration range $0.01-0.05 \mathrm{~mol} \%$ were similar at about 0.01 . The $\mathrm{CI} / \mathrm{CP}$ values for chain-breaking phenolic antioxidants were $0.001-0.006$ at similar concentrations of $0.01 \mathrm{~mol} \%$. The value for ME and MMI was about 10 times greater than that for phenolic compounds. The $n$ value for the former was about $0.2-0.4$, whereas that for the latter was 0.9-1.7. The $n$ value for phenolic compounds was about 5 times greater than that for ME or MMP. 
Table 3. Radical-scavenging activity for thiols, thiobarbituric acid derivatives and chain-breaking phenolic antioxidants.

\begin{tabular}{|c|c|c|c|c|c|c|}
\hline Additive & $\begin{array}{l}\text { Conc. } \\
\text { mol\% }\end{array}$ & $\begin{array}{c}\mathrm{IP}^{\mathrm{a}} \\
(\mathrm{min})\end{array}$ & $n^{\mathrm{b}}$ & $\begin{array}{c}\text { IRP }^{c} \\
(\% / \mathrm{min})\end{array}$ & $\left(\mathbf{k}_{\mathrm{inh}} / \mathbf{k}_{\mathbf{p}}\right)^{\mathbf{d}}$ & $(\mathrm{CI} / \mathrm{CP})^{\mathrm{e}}$ \\
\hline Experiment 1. Control & - & 0 & - & 1.30 & - & - \\
\hline \multirow[t]{4}{*}{ 2-Mercaptoethanol (ME) ${ }^{\mathrm{f}}$} & 0.01 & 1.27 & 0.4 & 1.30 & 60.65 & 0.01 \\
\hline & 0.01 & $0.81^{\mathrm{g}}$ & 0.3 & 1.34 & 94.0 & 0.01 \\
\hline & 0.05 & 4.63 & 0.3 & 1.28 & 16.82 & 0.01 \\
\hline & 0.05 & 4.12 & 0.3 & 1.33 & 18.18 & 0.01 \\
\hline Experiment 2. Control & - & 0 & - & 1.38 & - & - \\
\hline \multirow[t]{3}{*}{ 2-Mercapto-1-methylimidazole (MMI) } & 0.01 & 0.65 & 0.2 & 1.42 & 110.47 & 0.01 \\
\hline & 0.02 & 1.36 & 0.3 & 1.35 & 55.52 & 0.01 \\
\hline & 0.05 & 3.54 & 0.3 & 1.33 & 21.57 & 0.01 \\
\hline Experiment 3. Control & - & 0 & - & 1.25 & - & - \\
\hline 1,3,5-Trimethyl-2-thiobarbituric acid (1) & 1 & -2.06 & - & 1.36 & - & - \\
\hline 1 & 1 & -1.99 & - & 1.42 & - & - \\
\hline 1,5,5-Trimethyl-2-thiobarbituric acid (2) & 1 & 1.03 & 0.004 & 1.24 & 79.66 & 0.85 \\
\hline 5,5-Dimethyl-thiobarbituric acid (3) & 1 & 24.06 & 0.9 & 1.17 & 3.53 & 0.04 \\
\hline 5-Butyl-2-thiobarbituric acid (4) & 1 & 5.7 & 0.02 & 1.29 & 13.57 & 0.14 \\
\hline 5-Butylbarbituric acid (5) & 1 & -0.3 & - & 1.27 & 44.76 & 0.48 \\
\hline Experiment 4. Control & - & 0 & - & 1.23 & - & - \\
\hline 1 & 1 & -2.07 & - & 1.29 & - & - \\
\hline 5-Allyl-2-thiobarbituric acid (6) & 1 & 5.48 & 0.02 & 1.27 & 14.33 & 0.15 \\
\hline 5-Allyl-1,3-dimethyl-2-thiobarbituric acid (7) & 1 & 0.86 & - & 1.18 & - & - \\
\hline 5-(4-Vinylbenzyl)-2-thiobarbituric acid (8) & 1 & 5.35 & 0.02 & 1.37 & 13.62 & 0.15 \\
\hline Experiment 5. Control & - & 0 & - & 1.30 & - & - \\
\hline$p$-Cresol (4-Methylphenol) & 1 & 59.0 & 2.2 & 1.29 & 1.48 & 0.02 \\
\hline Eugenol (4-Allyl-2-methoxyphenol) & 1 & 79.0 & 2.0 & 1.08 & 1.17 & 0.01 \\
\hline Guaiacol (2-Methoxyphenol) & 1 & 53.8 & 2.0 & 1.26 & 1.48 & 0.02 \\
\hline Hydroquinone (1,4-Dihydroxybenzene) & 1 & 60.7 & 2.3 & 0.27 & 6.08 & 0.06 \\
\hline Phenol & 1 & 44.0 & 1.7 & 0.64 & 3.5 & 0.04 \\
\hline Thymol (6-Isopropyl-3-methylphenol) & 1 & 63.4 & 2.4 & 1.20 & 1.32 & 0.01 \\
\hline$p$-Cresol & 0.01 & 3.64 & 1.3 & 1.17 & 23.38 & 0.002 \\
\hline Eugenol & 0.01 & 2.68 & 0.9 & 1.25 & 12.68 & 0.001 \\
\hline Hydroquinone & 0.01 & 4.78 & 1.7 & 1.20 & 17.36 & 0.002 \\
\hline Phenol & 0.01 & 1.61 & 0.7 & 1.22 & 52.22 & 0.006 \\
\hline$\alpha$-Tocopherol & 0.01 & 4.5 & 1.7 & 1.20 & 18.11 & 0.002 \\
\hline
\end{tabular}

AIBN, $1.0 \mathrm{~mol} \%$; ${ }^{\mathrm{a}}$ Induction period; ${ }^{\mathrm{b}}$ Stoichiometric factor. The $n$ was calculated using Equation 1.

${ }^{\mathrm{c}}$ Initial rate of polymerization; ${ }^{\mathrm{d}}$ The ratio of the rate constant of inhibition and that of propagation calculated using Equation 4; ${ }^{\mathrm{e}}$ The ratio of the chain inhibition to that of chain propagation calculated using Equation 6; ${ }^{\mathrm{f}}$ The mean and standard error of IP values for 0.01 and $0.05 \mathrm{~mol} \%$ were $1.04 \pm 0.23$ and $4.38 \pm 0.25$, respectively; ${ }^{\mathrm{g}}$ Derived from Table 2 . The values of IP ${ }^{\mathrm{a}}$ and IRP ${ }^{\mathrm{c}}$ were the mean of two or three independent experiments and their standard error was $<5 \%$. Experiments 3 and 4 were taken from our data reported previously [4,5].

Next we investigated the radical scavenging activity for barbituric and thiobarbituric acid derivatives for compounds $\mathbf{1}-\mathbf{8}$ at a concentration of $1 \mathrm{~mol} \%$. The results are shown in Table 3.5 is 
barbituric acid, a reducing agent, and this showed no effect as an inhibitor. Thiobarbituric acid derivatives for $\mathbf{2}, \mathbf{3}, \mathbf{4}, \mathbf{6}$ and $\mathbf{8}$ showed the slightly longer IP than control's one. By contrast, compounds 1 and 7, particularly the former showed the lower IP than that of the control. The IP for $\mathbf{1}$ was approximately the half value of control. These finding suggested that $2, \mathbf{3}, \mathbf{4}, \mathbf{6}$ and $\mathbf{8}$ acted as a weak antioxidant, inhibitor, whereas $\mathbf{1}$ and $\mathbf{7}$ acted as a prooxidant. Note that a certain amount of atmospheric oxygen was contained in the sealed DSC sample pan, since it had been sealed in air. Therefore, polymerization curves for control broke when oxygen was completely consumed (Figure 1). Since purified MMA was used in the present study, no stabilizer was considered to be contained in a DSC sample pan. Therefore, no effect of a stabilizer such as 4-methoxyphenol on the polymerization of MMA may be considered. In this model, an alkyl radical $\left(\mathrm{R}^{*}\right)$ derived from the thermal decomposition of AIBN could react with dioxygen in a DSC sample pan to a peroxy radical (ROO*), consequently the possible retardation of polymerization for control occurred due to a certain amount of dioxygen resulting in the induction period with no additive. Thus, the IP value for the antioxidants tested was calculated from the difference of IP between antioxidants and control (Tables 3 and 4). The small IP value for $\mathbf{1}$ and $\mathbf{7}$, particularly the former has provoked interest in this model. The smaller IP for 1 than for control may be due to the finding that 1 reacted with dioxygen in the DSC container; the thiyl radical induced by radical oxidized 1 could react with dioxygen to form the thiol peroxy radical (RSOO*) in a DSC sample pan. Therefore, the negative value of IP for $\mathbf{1}$ and $\mathbf{7}$ may have been derived from their consumption of dioxygen in a DSC sample pan. On the other hand, the possibility that can not be completely excluded is that the small effect of $\mathbf{1}$ or $\mathbf{7}$ on the IP value may be due to their interaction of the surface of an alumina DSC sample pan because thiobarbituric acid derivatives showed a potent adhesion of resin to noble metals ( $\mathrm{Au}, \mathrm{Ag}, \mathrm{Pd})$ as well as base metals [7]. However, in the present study, $\mathbf{1}$ or $\mathbf{7}$ acted as a chain transfer agent for polymerization of MMA initiated by the thermal decomposition of AIBN (Table 4). Thus, the interaction of $\mathbf{1}$ and $\mathbf{7}$ with the surface of an alumina DSC sample pan may not be considered. Further studies may be necessary to clarify the mechanism of polymerization of MMA in the presence of these compounds using the present model.

By contrast, 3 showed an IP value of about $28 \mathrm{~min}$. Its $n$ value was calculated using Equation (1), and found to be about 0.9 . This suggested the formation of a dimer due to the radical-radical coupling reaction between 3 compounds, being estimated from the $n$ value of about 1. Compounds $\mathbf{2}, \mathbf{4}$ and $\mathbf{6}$ showed IP values of 5-9 min, indicating their weak antioxidant activity. Their $n$ value was markedly small, being within the range 0.004-0.02. By contrast, the $n$ value for chain-breaking phenols was approximately 2 , and similar to data reported previously [1,2]. The CI/CP value for all thiobarbituric acids used declined in the order $\mathbf{2}(0.85)>\mathbf{8}(0.15)>\mathbf{4}(0.14)>\mathbf{3}(0.04)$. By contrast, that for phenolic antioxidants was within the range $0.01-0.06$. The CI/CP values for $\mathbf{2}, \mathbf{4}$ and $\mathbf{8}$ were 10 times or greater than those for phenolic compounds. Barbituric acid (5) has an $n$-butyl substituent at the 5-position (R1 and R2 in Table 1), which is reported to act as a center for biological activity [12]. Thus, for thiobarbituric acid derivatives, the substituent at the 5 position may play an important role in the radical-scavenging activity. However, in addition to 5, compounds $\mathbf{6}, \mathbf{7}$ and $\mathbf{8}$ having an allyl substituent at the 5 position (R1) showed the small radical-scavenging activity. The radical scavenging activity for compounds 1 and 7, having a $\mathrm{CH}_{3}$ substituent at both $\mathrm{R} 3$ and $\mathrm{R} 4$ and an $\mathrm{H}$ substituent at R2, was compared with that of compounds 4, 6 and 8, having a $\mathrm{H}$ substituent at R2, R3 and R4 (see Table 1). A marked difference between the two groups was observed; the IP values for $\mathbf{1}$ and $\mathbf{7}$ were 
smaller than that of the control, whereas those for 4, 6 and 8 were larger than that of the control. From this it was considered that the radical-scavenging activity was highly influenced by the type of substituent at R3 and R4, but not by the alkyl substituent at R1, although these data were admittedly limited. Compound 3, which possesses a $\mathrm{CH}_{3}$ substituent at both $\mathrm{R} 1$ and $\mathrm{R} 2$ and an $\mathrm{H}$ substituent at both R3 and R4, was a potent inhibitor. There was a structure-property relationship for the function of both the type of substituent and its position within the molecule.

Next, we investigated the influence of thiobarbituric acid derivatives on the polymerization of MMA using our reported data [4,5] (Table 4). The average molecular weight (Mw) for $\mathbf{1}$, approximately $20 \times 10^{4}$, showed the smallest value, followed by 6 and 7 , at approximately $30-35 \times 10^{4}$. By contrast, compounds 2-5 and the control showed similar values at around $50 \times 10^{4}$ (Table 4). All compounds as well as the control showed a bimodal distribution that was relatively broad, with a molecular weight range of $\log 4$ to $\log 6.5$, whereas 1 showed a single modal value with a relatively narrow distribution. The polydispersity index for $\mathbf{1}$ was $2.2-2.7$, whereas those for compounds $\mathbf{2}-\mathbf{7}$ and the control were within about 3.2-3.5. From this it was considered that $\mathbf{1}$ may have acted as a chain transfer agent. Also, the KCL of $\mathbf{1}$ was about $10 \%$ greater than that of the control (Table 4), and $\mathbf{1}$ showed the highest conversion at the stage when the gel effect appeared among the test compounds, resulting from its higher IRP. The increase in KCL demonstrated for $\mathbf{1}$ may be due to the chain transfer reaction with MMA. However, the conversion (\%) of these compounds as well as the control during $100 \mathrm{~h}$ was almost uniform, being approximately $94 \%$.

Table 4. Effect of thiobarbituric acid derivatives on the average molecular weight of polymer formed during polymerization of MMA initiated by thermal decomposition of AIBN, based on reported data $[3,4]$.

\begin{tabular}{|c|c|c|c|c|c|c|}
\hline \multirow[b]{2}{*}{ Compound } & \multirow[b]{2}{*}{$\begin{array}{l}\text { IP } \\
\text { min }\end{array}$} & \multirow[b]{2}{*}{$\mathbf{R}_{\text {inh }} / \mathbf{R}_{\mathbf{p}}$} & \multicolumn{2}{|c|}{ Average molecular weight } & \multirow{2}{*}{$\begin{array}{l}\text { Polydispersity } \\
\text { index } \\
\text { Mw/Mn } \\
\end{array}$} & \multirow[b]{2}{*}{ KCL } \\
\hline & & & $\begin{array}{l}\left(\times 10^{4}\right) \\
\mathrm{Mn}\end{array}$ & $\begin{array}{l}\left(\times 10^{4}\right) \\
M w\end{array}$ & & \\
\hline \multicolumn{7}{|c|}{ A. Experiment 3.} \\
\hline Control & 0 & 1 & 14.6 & 49.4 & 3.4 & 315 \\
\hline 1 & -2.02 & 1.11 & 13.0 & 35.1 & 2.7 & 386 \\
\hline 2 & 1.03 & 0.99 & 15.4 & 53.1 & 3.5 & 312 \\
\hline 3 & 24.06 & 1.05 & 14.7 & 46.8 & 3.2 & 294 \\
\hline 4 & 5.7 & 0.94 & 14.5 & 46.6 & 3.2 & 325 \\
\hline 5 & -0.3 & 1.02 & 15.1 & 49.3 & 3.3 & 320 \\
\hline \multicolumn{7}{|c|}{ B. Experiment 4.} \\
\hline Control* & 0 & 1 & 14.6 & 49.4 & 3.4 & 315 \\
\hline 6 & 5.48 & 1.03 & 13.0 & 35.1 & 2.7 & 319 \\
\hline 7 & -0.86 & 0.96 & 12.5 & 33.3 & 2.7 & 297 \\
\hline 1 & -2.07 & 1.05 & 10.6 & 23.6 & 2.2 & 325 \\
\hline
\end{tabular}

For the IP and IRP values in experiments 3 and 4, see Table 2. Mn, number average molecular weight; $\mathrm{Mw}$, weight average molecular weight; $\mathrm{Rp}_{\mathrm{inh}} / \mathrm{Rp}_{\mathrm{con}}$, ratio of propagation in the presence of an inhibitor to that for the control; AIBN, 1 mol\%; Additive, 1 mol\%; MMA 9.4 mol/L. MMA was polymerized for $100 \mathrm{~min}$. The polydispersity index was calculated using Equation (6). KCL (kinetic chain length) was calculated using Equation (7). The value of $\mathbf{1}$ for A was the mean of two independent experiments and their standard error was $<7 \%$. *Taken from the experiment 3. 
The present method using DSC has been proven to be reliable for evaluating the activity of these compounds because DSC has high sensitivity [3,9]. Therefore, kinetic studies of compounds with very weak antioxidant activity can be performed. Since the $\mathrm{k}_{\mathrm{inh}} / \mathrm{k}_{\mathrm{p}}$ and CI/CP value for these compounds were dependent on their concentrations, the radical-scavenging activities for ME, MMI and phenolic antioxidants were compared at concentrations of $0.01-0.05 \mathrm{~mol} \%$, and also those for thiobarbituric acids and phenolic antioxidants were compared at a high concentration of 1 mol\%. We previously investigated the radical-scavenging activity for chain-breaking phenolic antioxidants using the induction period method in polymerization of MMA by thermal decomposition of benzoyl peroxide (PhCOO* benzoate radical) or AIBN ( ${ }^{*}$ alkyl radical) and it was found that polyphenols were a potent chain-breaking antioxidant $[13,14]$. Amorati et al. previously investigated the radical-scavenging activity of various phenolic compounds using the induction period method in the styrene system initiated by AIBN (ROO* peroxy radical) and it was found that their antioxidant activity was able to be kinetically evaluated using the induction period method [15].

Chemical adsorption of organic sulfur compounds on precious metal surfaces and assembly of organic thiols on these surfaces have been well investigated in the semiconductor industry [16,17]. Thiobarbituric acid compounds can promote adhesion of acrylate resin to precious metals used in dentistry [6]. MMI and some sulfur compounds have been reported to be absorbed on metal surfaces [18-20]. Also, thiobarbituric acid derivatives are expected to possess antioxidant, membrane protective, and radioprotective properties. Thus, organic sulfur compounds such as thiols and thiobarbituric acid compounds are of continuing interest.

\section{Conclusions}

The radical-scavenging activities of ME, MMI, (thio)barbituric compounds (1-8), and chain-breaking phenolic antioxidants ( $p$-cresol, eugenol, hydroquinone, phenol, thymol, and $\alpha$-tocopherol) were investigated using the induction period for polymerization of MMA initiated by AIBN. The CI/CP values for organic sulfur compounds, EM, MMI, 2, 4, 6 and 8 were about 10 times or more than those for the corresponding chain-breaking phenolic compounds. $\mathbf{3}$ acted as a potent inhibitor and with an $n$ value of approximately 1 , suggesting the formation of a dimer due to the coupling reaction of $\mathbf{3}$ compounds. The $\mathrm{Mw}$ for the polymers formed was the smallest for $\mathbf{1}$. The chain transfer reactivity for organic sulfur compounds was discussed.

\section{References}

1. Burton, G.W.; Ingold, K.U. Autoxidation of biological molecules. 1. Antioxidant activity of vitamin $\mathrm{E}$ and related chain-breaking phenolic antioxidants in vitro. J. Am. Chem. Soc. 1981, 103, 6472-6477.

2. Pryor, W.A.; Strickland, T.; Church, D.F. Comparison of the efficiencies of several natural and synthetic antioxidants in aqueous sodium dodecyl sulfate micelle solution. J. Am. Chem. Soc. 1988, 110, 2224-2229.

3. Kadoma, Y.; Kumada, W.; Asai, Y.; Sugita, Y.; Yokoe, I.; Fujisawa, S. Radical-scavenging activity of the reaction products of isoeugenol with thiol, thiophenol, mercaptothiazoline or mercaptomethylimodazole using the induction period. Molecules 2007, 12, 1836-1844. 
4. Kadoma, Y.; Imai, Y. Studies on initiator system for cold curing resin using thiobarbituric acid derivatives. J. Japan. Soc. Dent. Mater. Devices 1991, 10, 692-698.

5. Kadoma, Y.; Imai, Y. Visible-light initiator system using thiobarbituric acid with an allyl group. J. Japan. Soc. Dent. Mater. Devices 1992, 11, 430-435.

6. Kadoma, Y. Application of 5-(4-vinylbenzyl)-2-thiobarbituric acid to dental materials. J. Japan. Soc. Dent. Mater. Devices 1992, 11, 891-898.

7. O'Brien J.L.; Gornick, F. Chain transfer in the polymerization of methyl methacrylates. 1. Transfer with monomer and thiols. The mechanism of termination reaction at $60^{\circ}$. J. Am. Chem. Soc. 1955, 77, 4757-4763.

8. Karasu, F.; Arsu, N.; Yagci, Y. 2-Mercapto thioxanthone as a chain transfer agent in free-radical polymerization: A versatile route to incorporate thioxanthone moieties into polymer chain endes. J. Appl. Polym. Sci. 2007, 103, 3766-3770.

9. Fujisawa, S.; Kadoma, Y.; Yokoe, I. Radical-scavenging activity of butylated hydroxytoluene (BHT) and its metabolites. Chem. Phys. Lipids 2004, 130, 189-195.

10. Grusibic, Z.; Rempp, P; Benoit, H. A universal calibration for gel permeation chromatography. J. Polym. Sci. B5 1967, 5, 753-759.

11. Okada, Y.; Oono, Y. Polymerization of methyl methacrylates initiated by vitamin B12-formic acid, oxalic acid, or 2-mercaptoethanol system. NIHON KAGAKUKAISHI 1984, 105, 626-630.

12. Okada, J.; Esaki, T. C-13 NMR spectra of barbituric acid derivatives. Yakugaku Zasshi 1973, 93, 1014-1018.

13. Kadoma, Y.; Fujisawa, S. Radical-scavenging activity of dietary phytophenols in combination with co-antioxidants using the induction period method. Molecules 2011, 16, 10457-10470.

14. Fujisawa, S.; Kadoma, Y. Comparative study of the alkyl and peroxy radical scavenging activities of polyphenols. Chemosphere 2006, 62, 71-79.

15. Amorati, R.; Ferroni, F.; Pedulli, G.F.; Valgimigli, L. Modeling the co-antioxidant behavior of monofunctional phenols. Applications to some relevant compounds. J. Org. Chem. 2003, 68, 9654-9658.

16. Bain, C.D.; Troughton, E.B.; Tao, Y.T.; Evall, J.; Whitesides, G.M.; Nuzzo, R.G. Formation of monolayer films by the spontaneous assembly of organic thiols from solution onto gold. $J$. Am. Chem. Soc. 1989, 111, 321-335.

17. Nuzzo, R.G.; Dubois, L.H.; Allara, D.L. Fundamental studies of microscopic wetting on organic surfaces. 1. Formation and structural characterization of a self-consistent series of polyfunctional organic monolayers. J. Am. Chem. Soc. 1990, 112, 558-569.

18. Benali, O.; Larabi, L.; Traisnel, M.; Gengembre, L.; Harek, Y. Electrochemical, theoretical and XPS studies of 2-mercapto-1-methylimidazole adsorption carbon steel in $1 \mathrm{M} \mathrm{HClO}_{4}$. Appl. Surface Sci. 2007, 253, 6130-6139.

19. Ikemura, K.; Kojima, K.; Endo, T.; Kadoma, Y. Effect of the combination of dithiooctanoate monomers and acidic adhesive monomers on adhesion to precious metals, precious metal alloys and non-precious metal alloys. Dent. Mater. J. 2001, 30, 469-477. 
20. Shimoe, S.; Tanoue, N.; Satoda, T.; Nikawa, H.; Matsumura, H. Evaluation of single liquid primers with organic sulfur compound for bonding between indirect composite material and silver-palladium-copper-gold alloy. Dent. Mater. J. 2010, 29, 25-29.

(C) 2012 by the authors; licensee MDPI, Basel, Switzerland. This article is an open access article distributed under the terms and conditions of the Creative Commons Attribution license (http://creativecommons.org/licenses/by/3.0/). 